Original Article

\title{
Development and exploration of a Japanese version of the cerebral palsy quality of life for children questionnaire for primary caregivers: a pilot study
}

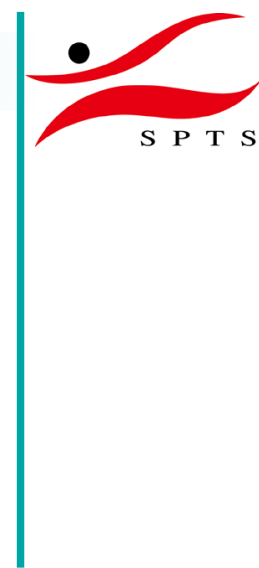

\author{
Tomoko Mutoh, MA ${ }^{1,2)}$, Tatsushi Mutoh, MD, DVM, PhD ${ }^{1)^{*}}$, Hiromi Kurosaki, PhD ${ }^{2)}$, \\ Hideo Shimomura, $\mathrm{MD}^{1)}$, Yasuyuki Taki, MD, $\mathrm{PhD}^{1)}$ \\ 1) Department of Nuclear Medicine and Radiology, Institute of Development, Aging and Cancer, \\ Tohoku University: 4-1 Seiryo-machi, Aoba-ku, Sendai 980-8575, Japan \\ 2) Division of Clinical Psychology, Graduate School of Human Sciences, Kobe Shoin Women's \\ University, Japan
}

\begin{abstract}
Purpose] We aimed to translate and validate a Japanese language version of the cerebral palsy quality of life for children questionnaire for primary caregivers and assess the relationship between quality of life of Japanese parents and their children's motor skills. [Participants and Methods] Fifty children (aged 4 to 12 years) and their parents (mothers) were enrolled. The parent-proxy version of the cerebral palsy quality of life for children questionnaire translated to Japanese was administered, and a validation study was performed using Cronbach's $\alpha$ as the key metric. The relationships between the parents' quality of life and children's Gross Motor Function Classification Scale levels were analyzed. [Results] We found that the age of the children and their parents and gender of the children were not significant factors affecting the quality of life domains. Significantly high values of internal consistency were detected among items within each quality of life domain, wherein Cronbach's $\alpha$ was between 0.72 and 0.89 . Two quality of life domains (Emotional well-being and Feeling about functioning) were significantly associated with Gross Motor Function Classification Scale levels. [Conclusion] Our data suggest that the original English version of the cerebral palsy quality of life for children questionnaire for primary caregivers was successfully translated to Japanese for use by Japanese-speaking parents caring for their children.

Key words: Cerebral palsy, Caregivers, Quality of life
\end{abstract}

(This article was submitted Apr. 18, 2019, and was accepted Jun. 15, 2019)

\section{INTRODUCTION}

Cerebral palsy (CP) is a permanent motor disability of multifactorial and complex etiologies, which are highly associated with public health issues even in the current era ${ }^{1)}$. Despite advances in fetal and maternal medical technologies, the prevalence of CP remains 2 to 2.5 per 1,000 live births in Japan ${ }^{2}$. The actual performance in the daily life of children with CP has been shown to be affected not only by their physical environment but also by numerous social and psychological factors.

The concept of "quality of life (QOL)" is an integrative definition of health-related well-being that takes into account a person's physical, psychological, and social dimensions ${ }^{3)}$. In terms of psychosocial properties of children with CP, it is increasingly accepted that a family-centered approach is best and that this requires the primary caregiver, usually the mother, to be an active participant in their child's care ${ }^{4}$. Most of these caregivers tend to have a poor QOL due to a sense of inferiority

*Corresponding author. Tatsushi Mutoh (E-mail address: tmutoh@tohoku.ac.jp) (Supplementary material: refer to PMC https://www.ncbi.nlm.nih.gov/pmc/journals/2193/)

(C2019 The Society of Physical Therapy Science. Published by IPEC Inc. 
or guilt, and they also often have a feeling of being isolated from their neighbors ${ }^{5}$. Therefore, it is important to detect, and help address, abnormalities in such a caregiver's QOL, in conjunction with their children's psychosocial development and functioning in society. Surprisingly, reliable tools to validly measure a caregiver's perception about their own health status, comfort, well-being, and ease of taking care of children with CP are currently limited ${ }^{6}$.

However, the CP QOL-Child is a condition-specific questionnaire designed for children with CP, to assess their well-being rather than their ill-being ${ }^{7)}$, and it includes a section aimed at caregivers of CP children between 4 and 12 years of age. The aim of this part of the instrument is to recognize the importance of obtaining the views of the primary caregivers of $\mathrm{CP}$ children, as these results can have material implications for the children ${ }^{8)}$. Although we believe the parent-proxy version of the CP QOL-Child is a well-developed measurement scale, it has not been translated nor has it been analyzed for its effectiveness regarding caregivers living in Japan.

Therefore, we conducted a pilot study to 1) translate and validate a Japanese language version of the CP QOL-Child questionnaire for primary caregivers, and to 2) assess the relationship between QOL in Japanese parents and their children's motor skills.

\section{PARTICIPANTS AND METHODS}

For this study, we developed a prospective, single-arm protocol that includes pairs of children with CP and their primary caregivers (i.e., parents or legal guardians). The eligible participants were recruited from various health care, recreation, and rehabilitation facilities in northern Japan between April 2017 and March 2019. This study was conducted in accordance with the Declaration of Helsinki and performed after approval by the Society of Physical Therapy Science Ethics Committee (Approval number: SPTS2016007). Written informed consent was obtained from each adult study participant.

Caregivers were asked to fill out questionnaires during visits to healthcare facilities (e.g., routine physical examination/ medication, physiotherapy, therapeutic horseback riding ${ }^{9-11)}$, and daycare or recreation centers), the questions of which address the child's age and gender as well as the caregiver's data including age, gender, and education. The child's latest Gross Motor Function Classification Scale (GMFCS) levels were measured simultaneously to assess their impact on various aspects of the caregiver's QOL.

Formal permission to translate the original English version of the primary caregiver proxy form into Japanese was obtained from the instrument's senior/corresponding authors. We then proceeded in six steps to develop and validate a Japanese version of the tool as follows: 1) The original English version was translated into Japanese by two translators separately, 2) For each English language sentence, we then selected the Japanese text from the two alternatives which most closely represented the intended meaning in the original, 3) A preliminary examination was executed by inviting three Japanese parents to fill out the draft version of the questionnaire, 4) Revisions of the problematic items were performed, 5) As a quality test, a backward translation into English by two independent translators was performed, and 6) Any material differences between the backward and original translations were reviewed and resolved. During each translation process, all authors of this study confirmed that the Japanese translated questionnaire was clear and easy to understand by Japanese-speaking parents. After carefully reviewing the final version, the present pilot study was conducted by a face-to-face interview, in accordance with CP QOL-Child Manual (http://www.cpqol.org.au/V2 m.\%20CPQOL\%20child\%20manual.pdf).

For analysis of psychometric properties, the primary caregiver CPQOL questionnaire [containing 66 items consisted of 9 categories (Family \& Friends, Communication, Health, Participation, Access to Services, Special Equipment, Pain and Bother, Some final questions about your child, and Your Health)] was grouped into seven large domains: 1) Social well-being \& acceptance (12 items), 2) Feelings about functioning (12 items), 3) Participation \& physical health (11 items), 4) Emotional well-being $\&$ self-esteem (6 items), 5) Access to services (8 items), 6) Pain \& the impact of disability (12 items), and 7) Family health (4 items) (Supplementary Table 1). These items are scored on a 9-point scale (range 1-9), except for one question in domain 6 , regarding pain \& the impact of disability, rated on a 5-point scale (range 1-5). All items were averaged per each domain and were transformed to a scale with a possible range of 0-100 as described in the CP QOL-Child Manual.

All analyses were performed using the "BellCurve for Excel" software (SSRI, Tokyo, Japan). The Mann-Whitney $U$-test was used to compare differences between non-normally distributed variables, and the Kruskal-Wallis rank sum test was conducted to examine the differences on each QOL domain according to the GMFCS levels, followed by Shirley-Williams' test for multiple pairwise comparisons. Shirley-Williams' multiple comparison test. Individual results were also elaborated using the Spearman test. Cross-tabulation $\left(\chi^{2}\right)$ tests were used for assessing independence between categorical variables [GMFCS levels and age (4-6 years, 7-9 years, and 10-12 years for children; twenties, thirties, and forties for adults) or gender (male and females)]. Cronbach's alpha coefficient was utilized to assess the reliability of the translated questionnaire, where values of this coefficient between 0.70 and 0.90 indicate high reliability. Data are presented as means \pm standard deviations (range) or number (\%) unless stated otherwise. The significance level was set at $\mathrm{p}<0.05$.

\section{RESULTS}

Demographic data for both children and their caregivers ( $\mathrm{n}=50$ each) is presented in Table 1. All the children had spastic type CP over bilateral lower limbs, and all questionnaires of the CP QOL were filled out by mothers. There were no signifi- 
Table 1. Demographic data of the studied Japanese participants

\begin{tabular}{|c|c|c|}
\hline \multicolumn{2}{|c|}{ Characteristics } & Frequency \\
\hline \multicolumn{3}{|c|}{ Children $(\mathrm{n}=50)$} \\
\hline \multicolumn{2}{|l|}{ Age (years) } & $9 \pm 3(4-12)$ \\
\hline \multicolumn{2}{|c|}{ Gender (male/female) } & $20 / 30(40 / 60)$ \\
\hline \multirow[t]{5}{*}{ GMFCS levels } & I & $10(20)$ \\
\hline & II & $13(26)$ \\
\hline & III & $15(30)$ \\
\hline & IV & $8(16)$ \\
\hline & $\mathrm{V}$ & $4(8)$ \\
\hline \multicolumn{3}{|c|}{ Primary caregivers $(n=50)$} \\
\hline \multicolumn{2}{|c|}{ Age (years) } & $36 \pm 6(25-47)$ \\
\hline \multicolumn{2}{|c|}{ Respondent (mother/father) } & $50 / 0(100 / 0)$ \\
\hline \multicolumn{2}{|c|}{ Education (College level or higher) (\%) } & $50(100)$ \\
\hline
\end{tabular}

Table 2. Descriptive data and reliability of psychometric properties of the Japanese CP QOL-Child questionnaire for primary caregivers

\begin{tabular}{lccc}
\hline \multicolumn{1}{c}{ Domain } & Averaged score* & Transformed scale & Cronbach's $\alpha$ coefficient $^{\#}$ \\
\hline Social well-being \& acceptance (12 items) & $5.8 \pm 0.7(1-9)$ & $64.8 \pm 7.9(11.1-100)$ & 0.886 \\
Feeling about functioning (12 items) & $5.2 \pm 0.9(2-8)$ & $57.3 \pm 9.9(22.2-88.9)$ & 0.720 \\
Participation \& physical health (11 items) & $5.2 \pm 0.6(1-9)$ & $57.5 \pm 6.7(11.1-100)$ & 0.752 \\
Emotional well-being \& self-esteem (6 items) & $5.2 \pm 0.4(3-8)$ & $57.6 \pm 4.6(33.3-88.9)$ & 0.729 \\
Access to services (8 items) & $5.2 \pm 0.6(3-8)$ & $57.3 \pm 7.0(33.3-88.9)$ & 0.796 \\
Pain \& the impact of disability (12 items) & $3.7 \pm 0.8(1-9)$ & $43.3 \pm 4.8(11.1-100)$ & 0.756 \\
Family health (4 items) & $5.4 \pm 0.7(1-9)$ & $59.9 \pm 7.5(11.1-100)$ & 0.771 \\
\hline
\end{tabular}

Values are expressed as mean $\pm \mathrm{SD}$ (range).

CP QOL-Child: cerebral palsy quality of life for children.

*Scored on a 9-point scale (range 1-9), except for one question of "Pain \& the impact of disability" rated on a 5-point scale (range 1-5). All items (Supplementary Table 1) were averaged per each domain and were transformed to a scale with a possible range of $0-100$, in accordance with the CP QOL-Child Manual.

\#Values between 0.70 and 0.90 indicate high reliability.

cant relationships between GMFCS levels and the gender or age of the children or the age of the mother ( $p>0.05)$.

In this study, all parents completed the questionnaires within 30 minutes, with less than 1\% missing data, except for 8 items of "Access to Services" and "Social well-being \& acceptance" (Supplementary Table 1), where approximately 40\% of the questions had missing data because many children had never used specialized equipment or support services. Therefore, to calculate the missing data index in a manner consistent across the entire questionnaire, it was necessary to remove three questions from this domain to compensate for the experience of many of the parents in this regard.

The overall QOL score and Cronbach's $\alpha$ for assessing the internal consistency of the questionnaire are shown in Table 2. For all QOL domains, Cronbach's $\alpha$ was scored between 0.72 and 0.89 . We further analyzed the relationships between the GMFCS levels of the children with the QOL domain values of the caregivers, as shown in Table 3. We found statistically significant differences between the GMFCS levels and two out of the seven CP QOL domains, namely "Emotional wellbeing" $\left(\chi^{2}=9.60 ; p=0.019 ; \mathrm{df}=4\right)$, and "Feeling about functioning" $\left(\chi^{2}=11.83 ; \mathrm{p}=0.048 ; \mathrm{df}=4\right)$. Both of these areas (using the averaged scores for each child) turned out to be negatively correlated with the child's GMFCS levels $[r=-0.58,95 \%$ confidence interval (CI): -0.82 to $-0.19 ; \mathrm{p}=0.007$ and $r=-0.48,95 \% \mathrm{CI}:-0.76$ to $-0.04 ; \mathrm{p}=0.034$, respectively].

\section{DISCUSSION}

To create an appropriate instrument for assessing the QOL of children with $\mathrm{CP}$, it is necessary to conduct studies on an international level. However, there had previously been no appropriate condition-specific metrics for caregivers in Japan working with CP children. As an alternative it has been possible to use the Japanese version of the somewhat generalized World Health Organization QOL-BREF (WHOQOL-BREF) ${ }^{12}$ ), which is aimed at assessing the QOL of primary family caregivers for patients with intractable and rare diseases ${ }^{13)}$. To the best knowledge of the authors, our study is the first attempt to translate the world-wide psychometric battery for testing the QOL of primary caregivers of CP children into Japanese.

According to a proposed psychometric protocol for evaluating medical scoring instruments (PPM-S; consisted of 11 questions), published as the Japanese Guidelines for the Rehabilitation of Cerebral Palsy, scales developed in other languages 
Table 3. Relationship between psychometric properties of the Japanese CP QOL-Child questionnaire for primary caregivers and GMFCS levels

\begin{tabular}{|c|c|c|c|c|c|c|}
\hline \multirow{2}{*}{ Domain } & \multicolumn{5}{|c|}{ GMFCS level } & \multirow{2}{*}{ p-value } \\
\hline & $\mathrm{I}$ & II & III & IV & $\mathrm{V}$ & \\
\hline Social well-being \& acceptance & $69.4 \pm 6.5$ & $63.2 \pm 6.6$ & $69.8 \pm 4.1$ & $59.7 \pm 16.4$ & $54.0 \pm 12.4$ & 0.18 \\
\hline Feeling about functioning & $64.3 \pm 4.6$ & $56.0 \pm 4.2^{*}$ & $58.4 \pm 3.4^{*}$ & $52.4 \pm 7.3^{*}$ & $49.6 \pm 4.5^{*}$ & 0.048 \\
\hline Participation \& physical health & $62.7 \pm 3.8$ & $55.0 \pm 4.1$ & $60.9 \pm 4.5$ & $56.2 \pm 11.7$ & $54.6 \pm 12.5$ & 0.30 \\
\hline Emotional well-being \& self-esteem & $67.3 \pm 2.8$ & $59.3 \pm 3.7^{*}$ & $59.0 \pm 5.8^{*}$ & $52.3 \pm 6.3^{*}$ & $50.6 \pm 2.8^{*}$ & 0.019 \\
\hline Access to services & $46.4 \pm 10.0$ & $58.3 \pm 6.8$ & $59.0 \pm 8.4$ & $51.5 \pm 12.6$ & $49.8 \pm 2.7$ & 0.32 \\
\hline Pain \& impact of disability & $59.9 \pm 4.2$ & $46.7 \pm 6.8$ & $43.3 \pm 15.1$ & $43.8 \pm 11.7$ & $28.0 \pm 9.2$ & 0.088 \\
\hline Family health & $61.1 \pm 14.7$ & $62.5 \pm 12.1$ & $62.0 \pm 12.0$ & $56.3 \pm 18.6$ & $51.9 \pm 9.8$ & 0.77 \\
\hline
\end{tabular}

Values are expressed as mean $\pm \mathrm{SD}$.

CP QOL-Child: cerebral palsy quality of life for children; GMFCS: Gross Motor Function Classification Scale for children with CP.

Each domain has a score with a possible range of 0-100 calculated in accordance with the CP QOL-Child Manual.

\#Values between 0.70 and 0.90 indicate high reliability.

Bold numbers indicate statistically significant differences $(\mathrm{p}<0.05)$ found among the five levels of GMFCS and each CP QOL domain (Kruskal-Wallis test).

$* \mathrm{p}<0.05$ vs. GMFCS I (Shirley-Williams’ post hoc test).

should be translated into Japanese, but only after they have undergone a rigorous confirmation of validity and reliability with appropriate experimental procedures in their language of origin (total PPM-S of $\geq 6$ ) ${ }^{14}$ ). To evaluate the reliability of this scale for native speakers of Japanese, we made best efforts to use the same evaluation procedures as those described in the original version (PPM-S=8). With regard to the internal consistency of the rating scale, the coefficients of Cronbach's $\alpha$ in the original CP QOL instrument for primary caregivers range between 0.74 and $0.92^{7)}$, while translated versions have been reported to vary from 0.78 to 0.92 (Chinese) $)^{15)}$ and from 0.61 to 0.87 (Persian) ${ }^{16)}$. With the Japanese version generating similar values of Cronbach's $\alpha$ (range: $0.72-0.89$ ), our results showed an excellent reliability of the test compared to both the original and to other translated versions.

In this study, using the new instrument, we found that the well-being status of functioning and emotion of the caregivers was significantly associated with the motor skills of the CP children, as estimated by the GMFCS. In addition, moderate levels of negative correlation could also be specified by these two domains. Interestingly similar results were obtained from validation studies regarding the CP QOL of Chinese and Persian caregivers $\left.{ }^{15}, 16\right)$. In fact, mild to moderate correlations ( $r>-0.2$ to -0.5 ) could be found between GMFCS and 3 QOL domains (functioning, emotional well-being, as well as participation and physical health) for both studies. These findings may support the expectation that the status of children asked by these items is relatively easy to identify and label for their parents.

The main limitation of this study is its relatively small number of participants. A second but less significant drawback is that we had only health care and/or recreation facility-based data, not information about the broader backgrounds and communities of the parents and CP children in this investigation, and this means that the possibility of a selection bias (e.g., regarding the age and education level of the parents) cannot be excluded. Since this is only a pilot study, the external validity of our results needs to be verified. Third, we did not gather data from children with CP by asking them to fill out and assess their own QOL. Therefore, this research area needs to be expanded to include a self-report questionnaire for children, to investigate whether there is a significant association between their results with those of their caregivers. Finally, the crosssectional nature of this study did not allow us to examine the performance of our translated version of the test over time. To further explore the reliability of our results and to determine whether our current findings hold up as CP children and their caregivers move forward in time, longitudinal studies using much larger populations would be highly productive.

Overall, the present data suggest that the original English language version of the CP QOL for primary caregivers was successfully translated into Japanese and that this new instrument can be validly used to assess the QOL of Japanese-speaking parents caring for their children with CP. Therefore, further clinical studies in Japan using this new localized version of the CP QOL would likely be quite productive in helping to evaluate the psychosocial QOL of such caregivers.

\section{Funding}

This work was supported by grants from the Society of Physical Therapy Science (H27) and JSPS KAKENHI Grant Number JP18K10773.

\section{Conflicts of interest \\ None.}




\section{ACKNOWLEDGMENTS}

The authors would like to thank all participants and staff members of healthcare facilities that were involved with this project. We are grateful to Dr. Hirokazu Tsubone (Graduate School of Agricultural and Life Sciences, The University of Tokyo) for continuous encouragement of this study. The translated version of the CP QOL-Child parent-proxy version (for parents of children aged 4-12 years) will be provided on the request of researchers or clinicians.

\section{REFERENCES}

1) Richards CL, Malouin F: Cerebral palsy: definition, assessment and rehabilitation. Handb Clin Neurol, 2013, 111: 183-195. [Medline] [CrossRef]

2) Hasegawa J, Toyokawa S, Ikenoue T, et al. Prevention Recurrence Committee, Japan Obstetric Compensation System for Cerebral Palsy: Relevant obstetric factors for cerebral palsy: from the nationwide obstetric compensation system in Japan. PLoS One, 2016, 11: e0148122. [Medline] [CrossRef]

3) Davis E, Shelly A, Waters E, et al.: Measuring the quality of life of children with cerebral palsy: comparing the conceptual differences and psychometric properties of three instruments. Dev Med Child Neurol, 2010, 52: 174-180. [Medline] [CrossRef]

4) Myrhaug HT, Jahnsen R, Østensjø S: Family-centred practices in the provision of interventions and services in primary health care: a survey of parents of preschool children with cerebral palsy. J Child Health Care, 2016, 20: 109-119. [Medline] [CrossRef]

5) Basaran A, Karadavut KI, Uneri SO, et al.: The effect of having a children with cerebral palsy on quality of life, burn-out, depression and anxiety scores: a comparative study. Eur J Phys Rehabil Med, 2013, 49: 815-822. [Medline]

6) Gilson KM, Davis E, Reddihough D, et al.: Quality of life in children with cerebral palsy: implications for practice. J Child Neurol, 2014, 29: 1134-1140. [Medline] [CrossRef]

7) Waters E, Davis E, Mackinnon A, et al.: Psychometric properties of the quality of life questionnaire for children with CP. Dev Med Child Neurol, 2007, 49: 49-55. [Medline] [CrossRef]

8) Waters E, Maher E, Salmon L, et al.: Development of a condition-specific measure of quality of life for children with cerebral palsy: empirical thematic data reported by parents and children. Child Care Health Dev, 2005, 31: 127-135. [Medline] [CrossRef]

9) Mutoh T, Mutoh T, Takada M, et al.: Application of a tri-axial accelerometry-based portable motion recorder for the quantitative assessment of hippotherapy in children and adolescents with cerebral palsy. J Phys Ther Sci, 2016, 28: 2970-2974. [Medline] [CrossRef]

10) Mutoh T, Mutoh T, Tsubone H, et al.: Impact of serial gait analyses on long-term outcome of hippotherapy in children and adolescents with cerebral palsy. Complement Ther Clin Pract, 2018, 30: 19-23. [Medline] [CrossRef]

11) Mutoh T, Mutoh T, Tsubone H, et al.: Effect of hippotherapy on gait symmetry in children with cerebral palsy: a pilot study. Clin Exp Pharmacol Physiol, 2019, 46: 506-509. [Medline] [CrossRef]

12) Tazaki M, Nakae Y: WHO QOL 26 Japanese version. Tokyo: Kanekoshobo, 1997.

13) Ihara H, Ogata H, Sayama M, et al.: QOL in caregivers of Japanese patients with Prader-Willi syndrome with reference to age and genotype. Am J Med Genet A, 2014, 164A: 2226-2231. [Medline] [CrossRef]

14) Kondo I: Psychometric property of medical evaluation score (PPM-S). In: Japanese guidelines for rehabilitation of cerebral palsy. The 2 nd edition. Tokyo: Kanehara \& Co., 2014, p 51.

15) Wang HY, Cheng CC, Hung JW, et al.: Validating the cerebral palsy quality of life for children (CP QOL-Child) questionnaire for use in Chinese populations. Neuropsychol Rehabil, 2010, 20: 883-898. [Medline] [CrossRef]

16) Soleimani F, Vameghi R, Kazemnejad A, et al.: Psychometric properties of the persian version of cerebral palsy quality of life questionnaire for children. Iran J Child Neurol, 2015, 9: 76-86. [Medline] 